\title{
Nearest Adjacent Node Discovery Scheme for Routing Protocol in Wireless Sensor Network
}

\author{
Manish R Tiwari, Nitika V Doohan, Sanjiv Tokekar \\ Department of Computer Science \& Engineering SDBCT, Indore, M.P., India \\ M.E. (CS), SDBCT Indore IET - DAVV, Indore, M.P., India
}

\begin{abstract}
The broad significance of Wireless Sensor Networks is in most emergency and disaster rescue domain. The routing process is the main challenges in the wireless sensor network due to lack of physical links. The objective of routing is to find optimum path which is used to transferring packets from source node to destination node. Routing should generate feasible routes between nodes and send traffic along the selected path and also achieve high performance. This paper presents a nearest adjacent node scheme based on shortest path routing algorithm. It is plays an important role in energy conservation. It finds the best location of nearest adjacent nodes by involving the least number of nodes in transmission of data and set large number of nodes to sleep in idle mode. Based on simulation result we shows the significant improvement in energy saving and enhance the life of the network.
\end{abstract}

Keywords: Energy Based Routing (EBR), LEACH, Nearest Adjacent Node Scheme, Shortest Path Routing Algorithm, WSNs

\section{INTRODUCTION}

Routing is a process of transferring packets from source node to destination node with minimum time for military emergency needs. Time factors may be the time elapsed of one route to another route of a router, network throughput of a link or link availability and reliability expressed as simple unit less numbers. Hence routing algorithm has to acquire, organize and distribute information about network states. It should generate feasible routes between nodes and send traffic along the selected path and also achieve high performance. Routing process uses a data structure called routing table at each node to store all the nodes which are at one hop distance from neighbour node. Here, computation of fitness for each node occurs respectively and bases on this select the path. It also stores the other nodes along with the number of hops to reach that node, followed by the neighbour node through which it can be reached. Router decides which neighbour to choose from routing table to reach specific destination.

Concurrent updates has occurs in shortest paths after a network change by distributing the network topology to all processors and using centralized Dijikstra's algorithm for shortest paths on every node [1]. If the topology of a network is represented as a weighted graph, where nodes represent processors, edges represent links between processors, and edge weights represent costs of communication among processors, then the typical update operations on a dynamic network can be modelled as insertions and deletions of edges and edge weight changes.

Thus while considering the problems of shortest path calculation \& energy efficient routing, the proposed novel approach of nearest adjacent node based shortest path routing algorithm (NAN-B-SPRA) based on extension of Dijkstra's algorithm is the best approach.

\section{BACKGROUND}

Network Routing is applied in a variety of applications, including military, transportation, telecommunications, etc. Network routing to direct vertex node to their destination in a dynamic traffic situation, with the aim of reducing the motoring time and to ensure and efficient use of available route resources is the main challenge in route discovery mechanism. For this efficient route discovery, we are using re-active protocols (on-demand protocols) which only start a route discovery procedure when needed. When a route from a source to a destination is needed, some sort of global search procedure is started. This does not require the constant updates being sent through the network, as in pro-active protocols, but it does cause delays, since the routes are not available and need to be found [2,3]. In some cases the desired route(s) are still in the route cache maintained by nodes. When this is the case there is no additional delay since routes do not have to be discovered.

\section{A. Co-Relation With Military Application of WSN}

In this proposed method, we deal with the issue of energy efficiency in wireless sensor networks for surveillance of a set of targets and critical information sending via shortest secure path for military application 
with known locality. Scenario of the network is chosen for armed forces purposes like surveillance of the boarder, battle fields and no go areas to acquire the information about enemies and their locations without taking the risk for human personal. We consider that a large number of sensors are distributed randomly in close proximity for monitoring and send the monitored information to a gateway node. All nodes are static and makes ad-hoc wireless sensor network. Every sensor nodes must monitor the area all the time in its operational range and each sensor has fixed transmission range. In network model we assume that each sensor has unique preconfigured Id and global/proactive routing algorithms are used. Main advantage of proactive algorithm is not route latency but drawback is the high maintenance overhead when many of the routes are never used [4]. Proactive routing is appropriate for networks with small size, low mobility and high communication rates. We proposed an algorithm called as NAN-B-SPRA for this purpose. By using NAN-B-SPRA we can find the perfect location of node for energy harvesting which also reduce the overall energy consumption and cost.

\section{B. Challenges in Shortest Path Routing}

Routing methods in WSNs have to deal with a number of challenges and design issues. Despite advancement in technology, sensor nodes in WSNs still have restrictions such as limited battery power, bandwidth constraint, limited computing power and limited memory. It creates the need for routing protocols to be highly adaptive and resource aware. Some of the challenges $[4,5,6]$ of routing protocol are:

1. Node deployment in either random or pre-determined manner.

2. Data reporting method which can be a time-driven, event-driven, query-driven or a hybrid of all of these methods.

3. Trade-off between energy consumption and accuracy of data gathered.

4. Node failure tolerance of the network.

5. Scalability, where routing method should be able to work with large networks.

6. Routing method should be adaptive for mobile sensor nodes.

7. Should support data aggregation to reduce redundant data.

\section{Energy Based Routing Protocol in WSN}

According to [7] Low Energy adaptive clustering hierarchy (LEACH) is a popular energy efficient adaptive clustering algorithm that forms node clusters based on the received signal strength. The cluster head $(\mathrm{CH})$ aggregates the sensed data from all transmits it to the BS. LEACH assumes that the base station is immobile and is located far from the sensors. All nodes are capable of communicating with the BS directly. The cluster head $(\mathrm{CH})$ can perform data aggregation and data dissemination. In LEACH the nodes form local clusters with one of the nodes acting as a local sink or cluster head. If the same node would remain as the cluster head throughout the working of the network, it would die quickly because of the extensive load from the participating sensors in the cluster. Hence the rotation of the cluster head in every round is necessary to distribute the load uniformly. Further energy dissipation can be reduced by aggregating the data from various sensor nodes at the cluster head. The operation of LEACH is broken up into rounds where each round begins with a setup followed by a steady state phase. The set up phase follows the above mentioned sequence

\section{RELATED STUDY}

A number of dynamic solutions for the shortest paths problem have been proposed in the literature also in the distributed case. Many research works have used explicitly or implicitly the SPT construction in WSN [8]. Depending on the routing strategy, these works propose an adequate link cost definition. In the basic approaches, the aim was to minimize the hop count towards the sink.

In this paper the author[8] provide a decremental and an incremental solution that are able to concurrently update shortest paths. The details of their contribution can be summarized as follows:

1. They propose a new decremental algorithm partially dynamic algorithm that is robust since it works in one phase (thus avoiding the concurrent updates of paths of previous work). Furthermore, it is able to concurrently update shortest paths in the case of multiple weight increase and delete operations. But the algorithm requires large space per node and can suffer of the looping phenomenon. However, the solution has been shown to be experimentally efficient when compared with two different implementations of the classical Bellman-Ford method.

2. They propose an extension of the incremental algorithm for weight decrease and insert operations that work also in the concurrent case, within the same bounds, that is O.maxdeg messages per operation and O.n space per node. Here, is the number of nodes affected by a set of weight decrease/insert operations. This is only a factor maxdeg far from the optimal incremental solution. Besides being theoretically efficient, this algorithm has been shown to be also experimentally fast [9].

In the paper [10], author uses the distance between any two nodes as an estimate for the transmission power required to send a packet between the two nodes and the propagation delay between them. The 
transmission energy required to send a bit has been found to have a distance dependence which is well modelled by $d n$, where $d$ is the distance between the transmitter and the receiver antennas and the exponent $n$ is determined from field measurements and the particular system at hand. Moreover, the propagation delay is directly proportional to the distance and related to it with the speed of signal propagation in the wireless medium. By constraining the maximum transmission distance that the wireless transmitter of each sensor node can reach, we can change the interconnection between the nodes and thus obtain different network topologies. In this, they model the routing problem as a single-sink unicast routing problem from the sensor nodes to the gateway taking our cost metric as the number of hops. This problem can be solved by any shortest-path routing algorithm. It is important to mention here that without using a constraint on the maximum transmission distance, the minimum number of hops routing algorithm reduces to the direct routing algorithm, in which each node sends its data directly to the gateway, as we assume that all the nodes can reach the gateway directly using their wireless transceivers [11].

Disjoint multi-path routing scheme with secret sharing is widely recognized as one of the effective routing strategies to ensure the safety of information. This kind of scheme transforms each packet into several shares to enhance the security of transmission. However, in many-to-one WSNs, shares have a high probability to traverse through the same link and to be intercepted by adversaries. In this paper, we formulate secret sharing based multi-path routing problem as an optimization problem. Here, M. A. Khan et. al. [12] aims at maximizing both network security and lifetime, subject to the energy constraints. To this end, a three-phase disjoint routing scheme, called Security and Energy-efficient Disjoint Route (SEDR), is proposed. Based on secret sharing algorithm, the SEDR scheme dispersive and randomly delivers shares all over the network in the first two phases, and then transmits these shares to the sink node. Both theoretical and simulation results demonstrate that our proposed scheme has significant improvement in network security under both scenarios of single and multiple black holes without reducing the network lifetime.

\section{NEAREST ADJACENT NODE SCHEME}

\section{A. Proposed Nearest Adjacent Node Based Shortest Path Routing Algorithm (NAN-B-SPRA)}

Shortest path routing algorithm or link state routing is the approach to find the shortest path for each node in the wireless sensor network. Calculate all the possible shortest paths for each node. Then find the NAN node (Figure 1). A node which is most nearest to the gateway sink node. Select a node which is used in maximum number of shortest paths

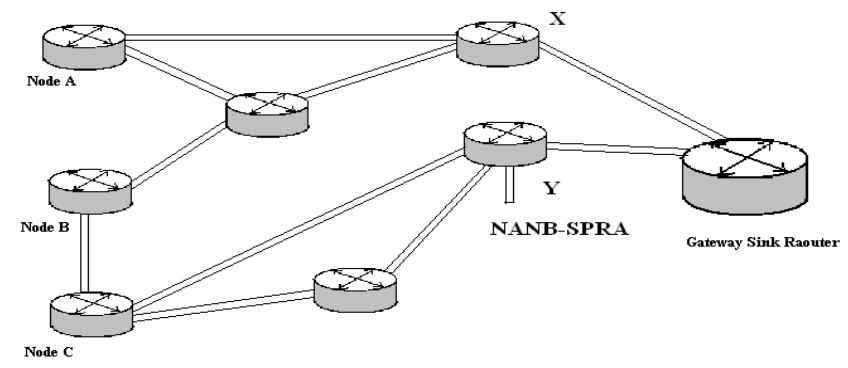

Figure1: Location of selected Nearest Adjacent Node (NAN)

In above network model, we assumed that sensed information is equally probable for all the nodes. Then we calculate the shortest path for the nodes A, B and C. Then we find out the nodes which are most nearest to the gateway node. In above network model, there are only two nodes $\mathrm{X}$ and $\mathrm{Y}$ which are closer to the gateway node. Then for selection, we give the preference to the node which is mostly used in the shortest paths. In above model, $\mathrm{Y}$ is node which is mostly used in all shortest paths. If nodes A, B and C transmit their data the entire time node $\mathrm{Y}$ will be included in their path. Then every node keeps its routes information towards the node Y for future communications.

Proposed algorithm finds the nearest adjacent node (NAN) in the network, that node should be active all the time while other nodes nearby it remain in sleep mode and keep sensing. As we use proactive routing, so each sensor knows its path towards the NAN node. Every node send its information to all the neighbor nodes through a wakeup message by which the initial states of the nodes gets activated. When NAN nodes receive the information it will forward the data to the gateway and sets all the other nodes into sleep mode. The critical issue in this solution is that if the actual node i.e. NAN remains active all the time then its energy source will be empty soon. The work resolves this issue by using the energy harvesting concept at NAN node. It also uses secondary battery which is rechargeable and coupled with photovoltaic cell. All the decision of routing must do at gateway sink node. 
Rerouting is the process to discover a route due to various circumstances where route is lost.

To take the decision for the gateway to perform rerouting is based on the following three

1. Sensor reorganization: The gateway may perform rerouting if an event happens that requires the reselection of active sensors.

2. Nodes' Battery Energy Level: The gateway may perform rerouting if the battery level of any active node drops to a certain level.

3. Energy Model Adjustment: it also occur after receiving an updated status from the sensors. Changes to the energy model might affect the optimality of the current routes, and thus new routes have to be generated.

All the nodes can generate energy from light, vibration, heat etc. but it will increase the system cost. We don't need to replace all the nodes with secondary sources. By replacing only one node (NANB node) resolves the issue and slightly increases the cost of the overall system. But effectively prolong the life time of sensor network. A solution can be more energy efficient by implementation of proposed algorithm with every new location of mobile base station. Split the network in equal parts and periodically change the position of base station in each part. Base station can be easily implemented at the place of NANB node in each part of the network instead of replacing it outside the network. NANB node will reduce the multi hop and number of transmission which directly reduce the energy consumption.

\footnotetext{
Algorithm

1. The router first computes the all possible paths $\&$ then calculates their respective weights.

2. The router then builds a status record for each nearest adjacent node based (NANB) on the network. The record contains the following fields:

$>\quad$ Predecessor field - shows the previous node.

$>\quad$ Length field - shows the sum of the weights from the source to that node.

$>\quad$ Label field - shows the status of node; each node have one status mode: "permanent" or "tentative."

3. Router then checks for the most adjacent node to gateway.

4. In the next step, the router initializes the parameters of the routing table record (for all nodes) and sets their label to "provisional" and their length to "infinity".

5. During this step, the router sets a T-node. If R1 is to be the source T-node, for example, the router changes R1's label to "permanent." Once a label is changed to "permanent," it never changes again.

6 . Find the most used shortest path from gateway.

7. The router updates the status record for all provisional nodes that are directly linked to the source T-node.

8. The router goes over all of the provisional nodes and chooses the one whose weight to R1 is lowest. That node is then the destination T-node.

9. If the new T-node is not R2 (the intended destination), the router goes back to step 5 .

10. If this node is $\mathrm{R} 2$, the router extracts its previous node from the status record and does this until it arrives at $\mathrm{R} 1$. This list of nodes shows the best route from R1 to R2 as shown in Figure. 2

The mentioned objectives are achieved by modifying the existence route discovery mechanism via Dijkstra's to some of its newer versions with the help of nearest node discovery. Proposed work also added an additional energy efficiency mechanism based on LEACH protocol to this approach to get more accurate \& well performed results in future. We will simulate the mechanism \& verify its results via NS-2 simulator.
}

\section{B. Future Benefit Metrics}

In future the performance of new version NANB-SPRA is measured in terms of following metrics evaluated:

Time to network partition: Defined as the time for the first node to die in the network.

Time for last node to die: This metric, along with the time to network partition metric, gives an indication of network lifetime.

Average and standard deviation of nodes' lifetime: This also gives a good measure of the network lifetime. A routing

algorithm, which minimizes the standard deviation of nodes' life, is predictable and thus desirable.

Average delay per packet: Defined as the average time a packet takes from a node in the sensing state to the gateway.

Network Throughput: Defined as the rate of data packets received at the gateway.

Average energy consumed per packet: This metric represents the average energy consumed in transmitting, and receiving a data packet. A routing algorithm that minimizes the energy consumed per packet will, in general, yields better energy savings and increased network lifetime. 


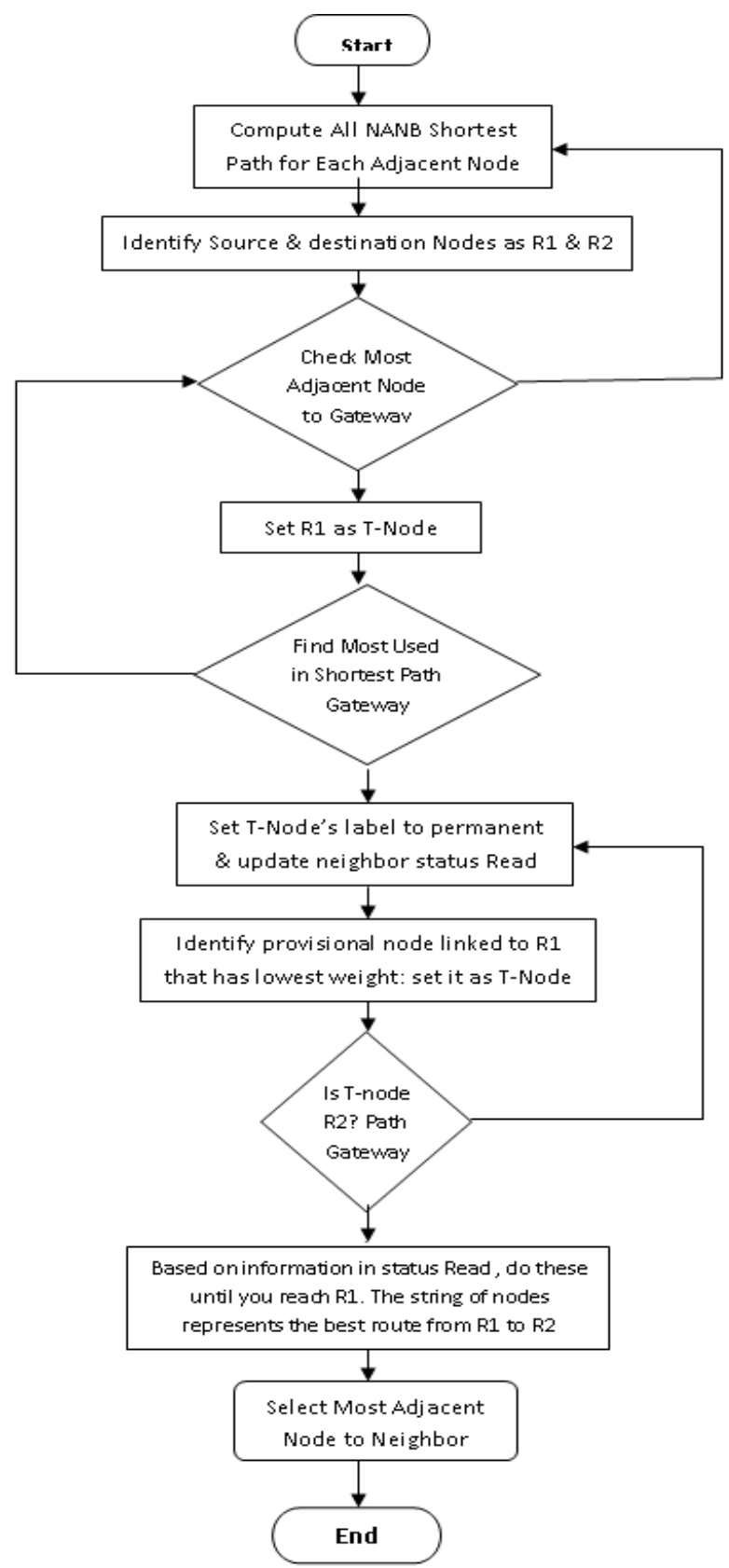

Figure 2: Process Flow of NANB-SPRA based Modified Dijkstra's algorithms

\section{IMPLEMENTATION AND RESULT ANALYSIS}

The proposed scheme is implemented using ns-2 tool and result analysis on the basis of following parameters-

Packet Delivery Ratio: Packet delivery ratio measured by calculating total no of received packets divided by total no of packets sent multiplied by hundred.
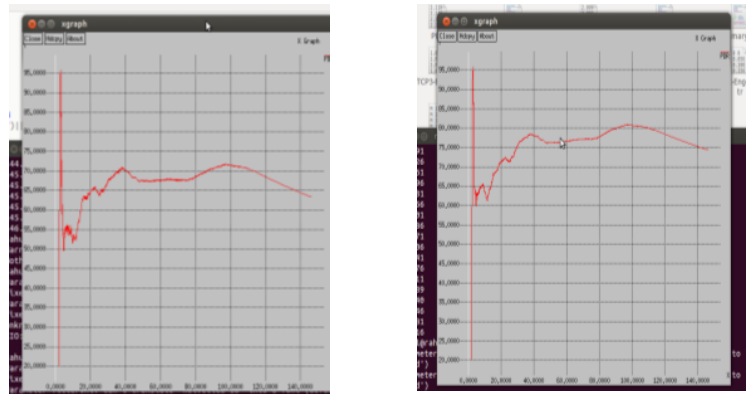
Figure 3: Packet Delivery Ratio

Throughput: Throughput of network determined by no of packets delivered at specific time unit.
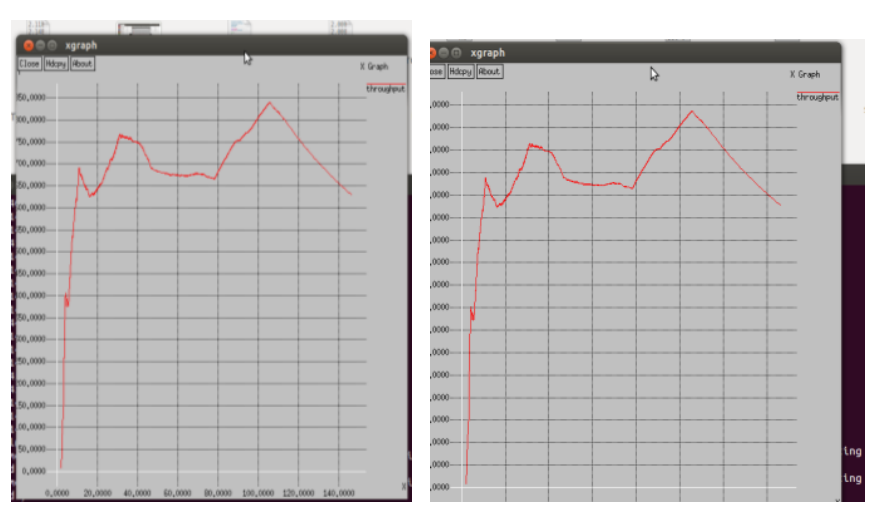

Figure 4: Throughput

Routing load: Routing-load calculated as total no of routing packets send and forward by nodes in the network.
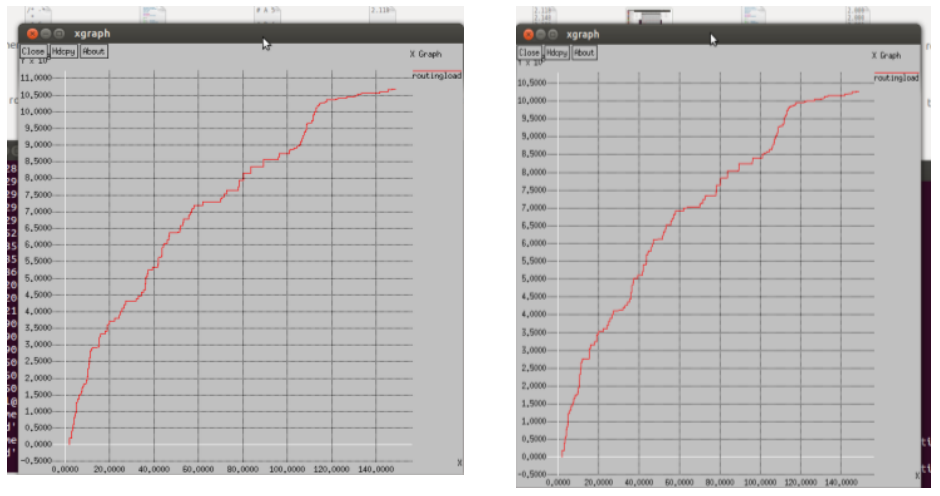

Summary

Figure 5: Routing load
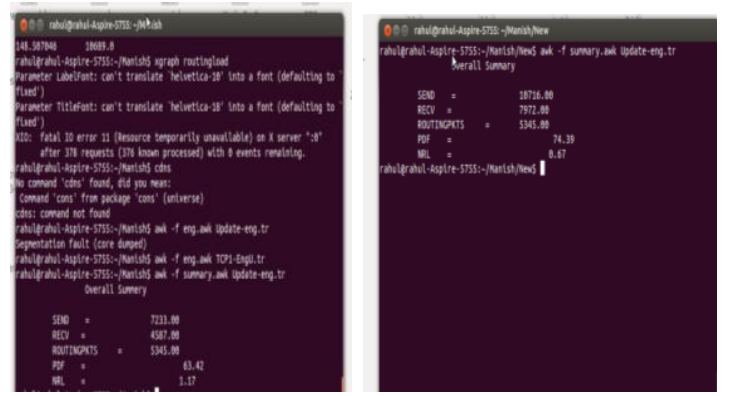

Figure 6: Summary

\section{CONClusion}

The proposed work on nearest adjacent node based shortest path routing (NANB-SPRA) routing algorithm is used to reduce the energy utilization in wireless sensor networks via efficient path. Implementation phase of this work will shows that the proposed extension performs well with respect to routing overhead incurred, while serving to minimize path stretch relative to optimality. Our algorithm involves least number of nodes during transmission and keeps one node active in sleep mode based on Dijkstra's having energy efficiency which makes use of continuous route optimization process. That significantly reduces the energy consumption during the transmission and sleep mode when there is no activity. An open research issue is the heterogeneity of energy resources of the nodes that must be resolved after practical implementation in any network. In our solution there is uneven energy consumption due to the topology of the network and nature of data flow. But that uneven energy consumption is helpful to reduce the energy consumption of entire network 


\section{FUTURE WORK}

As a future work, improvement in the proposed scheme can be accomplished in terms of both control traffic incurred and resulting gain in path optimality. Indeed, the proposed scheme detects the shortcuts, if any, only between the nodes which are located 2-hop away to each other. Therefore, as a next step, we would like to develop a route optimization scheme which is able to detect shortcuts between any pair of nodes on a connection. Finally, such a route optimization scheme can lead military official for effective transmission of emergency messages in very less span of time via without worrying about the path optimality, which has been addressed as a problem in local recovery operations.

\section{REFERENCE}

[1] S Cicerone, G D'Angelo, G D.Stefano \& D Frigioni "Partially dynamic efficient algorithms for distributed shortest paths" in Elsevier Journal of Theoretical Computer Science 411 (2010)

[2] M A. Youssef, M F. Younis, and K A. Arisha "A Constrained Shortest-Path Energy-Aware Routing Algorithm for Wireless Sensor Networks" in Honeywell International Inc Research Lab in 2009.

[3] Anfeng Liu \& Chao Zhang "Secure and Energy-Efficient Disjoint Multi-Path Routing for WSNs" in IEEE 2011

[4] J Gao, Q Zhao, W Ren, A Swami "Dynamic Shortest Path Algorithms for Hyper graphs" in Army Research Laboratory Network Science CTA under Cooperative Agreement W911NF-09-2-0053.

[5] S Yoon, R Dutta, M L. Sichitiu "Power aware routing algorithms for wireless sensor networks" in North Carolina State University Raleigh, NC 27695-7911

[6] A Liuyz, Z Zhengz, C Zhangy, Z Cheny, and X Shenz, "Secure and Energy-Efficient Disjoint Multi-Path Routing for WSNs" in IEEE 2011.

[7] M A. Youssef, M F. Younis, K A. Arisha "A Constrained Shortest-Path Energy-Aware Routing Algorithm for Wireless Sensor Networks" in Honeywell International Inc Dec 2011.

[8] S K .Singh, M P Singh, and D K Singh "Routing Protocols in Wireless Sensor Networks -A Survey" in IJCSES Vol.1, No.2, November 2010

[9] A. Rajeswari, P. T. Kalaivaani “ A Novel Energy Efficient Routing Protocols for Wireless Sensor Networks Using Spatial Correlation Based Collaborative Medium Access Control Combined with Hybrid MAC Network Protocols and Algorithms" ISSN 1943-3581 2011, Vol. 3, No. 4

[10] Z Bilgin B Khan "A Dynamic Route Optimization Mechanism for AODV in MANETs" in IEEE 2010

[11] N Zhang and J H Zhang "A Self-adapted Anycast Routing Algorithm Based on Mobile Agent in Wireless Sensor Network" in Journal of networks, vol. 6,no. 2, feb 2011

[12] M. A. Khan, M. Ahsan, G. A. Shah and M Sher "Multicast Routing Protocols in Wireless Sensor Networks (WSNs)" in journal of computing, vol 4, issue 9, Sep 2012, ISSN (Online) 2151-9617

[13] N.Pushpalatha and B.Anuradha "A Comparative Analysis of WSN Sensors Positioning Method using Iterative Routing Algorithm with Conventional Methods" in International Journal of Computer Applications (0975 - 8887) Volume 53- No.7, September 2012

[14] N A. S. Alwan, I K. Ibraheem \&, S M. Shukr "Fast Computation of the Shortest Path Problem through Simultaneous Forward and Backward Systolic Dynamic Programming “ in International Journal of Computer Applications (0975 - 8887) Volume 54- No.1, September 2012

[15] A G Delavar, A Alirezaie \& A A Baradaran "KGAWSN: An Effective Way to Reduce Energy Consumption in Wireless Sensor Networks by Kmeans and Genetic Algorithms" in International Journal of Computer Applications (0975 - 888) Volume 48No.12, June 2012

[16] A Norouzi1, F S Babamir, A H Zaim “A Novel Energy Efficient Routing Protocol in Wireless Sensor Networks" in Science research, Dec 2011. 